\title{
Bell Locality and the Nonlocal Character of Nature
}

\author{
Travis Norsen \\ Marlboro College \\ Marlboro, VT 05344 \\ norsen@marlboro.edu
}

(Dated: July 25, 2018)

\begin{abstract}
It is demonstrated that hidden variables of a certain type follow logically from a certain local causality requirement ("Bell Locality") and the empirically well-supported predictions of quantum theory for the standard EPR-Bell setup. The demonstrated hidden variables are precisely those needed for the derivation of the Bell Inequalities. We thus refute the widespread view that empirical violations of Bell Inequalities leave open a choice of whether to reject (i) locality or (ii) hidden variables. Both principles are indeed assumed in the derivation of the inequalities, but since, as we demonstrate here, (ii) actually follows from (i), there is no choice but to blame the violation of Bell's Inequality on (i). Our main conclusion is thus that no Bell Local theory can be consistent with what is known from experiment about the correlations exhibited by separated particles. Aside from our conclusion being based on a different sense of locality this conclusion resembles one that has been advocated recently by H.P. Stapp. We therefore also carefully contrast the argument presented here to that proposed by Stapp.
\end{abstract}

Key Words: EPR, Bell's Theorem, non-locality, Stapp, hidden variables

\section{INTRODUCTION}

Bell's Theorem [1] has given rise to a unique situation in science. Despite being hailed from all quarters as fundamentally important ("the most profound discovery of science," says H. P. Stapp [2]), the meaning of the theorem - what, exactly, it proves - remains widely and hotly contested. There are (at least) two camps. In the first camp are those who believe that Bell's Theorem proves that non-locality is a fact of nature, which must be reflected in any adequate theory. [1, 3, 4, 5, 6] Then there are, in the second camp, those who believe that Bell's Theorem proves only the impossibility of empirically viable local "hidden variable" alternatives to orthodox quantum theory. 7, 8]

It is widely accepted (by people in both camps) that two assumptions - locality and hidden variables - are needed in the derivation of the Bell Inequalities. Those in the first camp, however, frequently cite the EPR argument 9] as providing a link between these two principles: the existence of hidden variables, it is argued, follows from the requirement of locality. For example, Bell writes that

\footnotetext{
"My own first paper on this subject [Bell's Theorem] ... starts with a summary of the EPR argument from locality to deterministic hidden variables. But the commentators have almost universally reported that it begins with deterministic hidden variables." [1, pg 157]
}

Those in the second camp have either failed to understand the role of the EPR argument as part-one of Bell's two-part argument for non-locality, or have (following Bohr and his supporters) rejected the EPR argument as invalid.
The main purpose of the present paper is to present a new version of the EPR argument (from locality to deterministic hidden variables, the very hidden variables needed to then derive a Bell Inequality) which removes these objections and hence solidifies the viewpoint of the first camp. The argument to be presented differs in several important ways from the original EPR argument. First, instead of merely attempting to prove the incompleteness of the orthodox (wave-function-only) description of quantum states, we provide an actual constructive proof of the existence of particular hidden variables (that is, their required existence under the assumption of local causality). Second, in place of EPR's somewhat obscure locality assumption, we utilize Bell's mathematically precise definition of local causality (hereafter "Bell Locality"). This locality condition has been widely misunderstood and misrepresented in the Bell literature, so we discuss and defend it in some detail. The present paper thus completes the logically rigorous demonstration that empirical violation of Bell's Inequalities (under appropriate, delayed-choice conditions) signals the violation in Nature of Bell Locality. In short, we remove the basis (objections to EPR) of the second camp's arguments against the first camp.

Why is this debate between camps one and two even important? Because the life or death of the hidden variables program (advocated by those such as Einstein, Bohm, and Bell who were dissatisfied with the Copenhagen quantum theory) hangs in the balance. Those in the first camp argue that, since non-locality is a real fact about the physical world, physicists should remain open to explicitly non-local alternatives to orthodox quantum theory such as Bohmian Mechanics. [10] On the other hand, those in the second camp use Bell's Theorem against the hidden variables program, by arguing that any such alternatives to orthodox theory must con- 
flict with relativity and hence needn't be seriously considered.

This standard argument of the second camp against the first was clearly articulated by H. P. Stapp. Contrary to the view of those in the first camp, he writes, Bell's Theorem "shows only that if certain predictions of quantum theory are correct, and if a certain hiddenvariable assumption is valid, then a locality condition must fail. This locality condition expresses the physical idea, suggested by the theory of relativity, that what an experimenter freely chooses to measure in one spacetime region can have no effect of any kind in a second region situated spacelike relative to the first." Therefore, "the most natural conclusion to draw [from the empirical violation of Bell's Inequality] is not that locality fails, but rather that the hidden-variable assumption is false." After all: "Bell's hidden-variable assumption was an ad hoc assumption that had no foundation in the quantum precepts. Indeed it directly contradicted the quantum precepts." 11]

This is a widely held view whose implications for the hidden-variables debate were eloquently captured by N. David Mermin: "To those for whom nonlocality is anathema, Bell's Theorem finally spells the death of the hidden-variables program." [8]

In an interesting recent series of papers H. P. Stapp has questioned the reasoning behind this conclusion and presented arguments in support of the first camp. 11, 12, 13] Stapp's re-thinking is based on his attempt to prove a stronger version of Bell's Theorem which dispenses altogether with the assumption of hidden-variables. (Actually, Stapp's argument is based on Hardy's scenario 14] in which a simple logical contradiction - rather than an inequality - is deduced from the assumption of local hidden variables and quantum theory's probabilistic predictions. But this distinction is not relevant here.)

We believe, however, that Stapp's arguments for the first-camp position are fatally flawed. So, by way of clarifying the content of the first-camp-supporting arguments to be presented here, we shall contrast these with the arguments of Stapp. Let us then briefly review the logical structure of Stapp's attempts, to see how his approach differs from the approach of Bell (elaborated and completed in the present paper).

Using a certain locality principle, the predictions of quantum theory for the Hardy scenario, and an assumption about which experiment was freely chosen in the left $(L)$ wing of the experiment, Stapp establishes the truth of a certain statement (call it $S$ ) which refers to possible measurements and their outcomes on the right $(R)$. He then proves that, had the experimenter in region $L$ made a different free choice, $S$ would cease to be true. Therefore, the truth status of a statement pertaining exclusively to region $R$ depends on a free choice made in region $L$, which we may assume to be space-like separated from $R$. According to Stapp, this constitutes a kind of non-local action at a distance, and thus establishes that not merely empirically viable hidden-variable theories but, rather, any theory in agreement with quantum theory's predictions, must violate locality. It establishes, says Stapp, the ineliminable non-local character of quantum theory itself.

Stapp's argument, however, has been roundly criticized. The trouble pertains to Stapp's definition of locality, which definition William Unruh summarizes as follows: "the truth of a statement pertaining exclusively to possible events in region $R$ cannot depend on which free choice is to be made by the experimenter in region $L$." 15] As Mermin has clarified, this definition encompasses two very different "locality" ideas: first, that what an experimenter on one side chooses to measure can have no influence on the actual outcome of an actually-performed measurement on the other side; and second, that what an experimenter on one side chooses to measure can have no influence on what can be validly inferred about what would have happened had some other choice been made on the other side. 18] Violation of the first sort of locality would indeed appear to be in conflict with relativity's prohibition on superluminal causation; but it is only the latter (which seems unproblematic from the point of view of relativistic causality) that is established by Stapp's argument. For Stapp's statement $S$ is not merely a statement about the actual result of an actual experiment in $R$; it is rather a counterfactual conditional asserting that a certain outcome would have been obtained had a different measurement been made, given that, in fact, the actual experiment was performed and had a certain particular outcome.

The main objection of Mermin and others is not simply that the statement $S$ is a counter-factual conditional and hence not directly observable. The critics accept the validity in principle of such counterfactual statements, but point out that, although the counterfactual statement $S$ may appear superficially to "pertain exclusively to region $R$ ", it nevertheless refers implicitly to region $L$ via the fact that our grounds for accepting $S$ include statements explicitly pertaining to $L$. (The precise sense in which the meaning of $S$ includes implicit reference to region $L$ is made particularly clear in the essays of Shimony and Stein. [16, 17] ) As Mermin explains, Stapp's conclusion "does not mean ... that a choice made [on the left] can influence events [on the right]. What it does mean is that a choice made [on the left] can influence statements about events [on the right] that might have happened but did not.... The choice of experiment on the left cannot affect what actually happened ... on the right. But it can affect the kinds of inferences one can make about hypothetical behavior on the right." [18] And elsewhere: "because the influence [which Stapp's argument actually establishes] is only on the possibility of satisfying Stapp's criterion for the valid use of counterfactuals, it is inappropriately characterized as ... 'non-local'." 19]

We believe the various criticisms of Stapp's arguments are correct. But as already indicated, we also believe that Stapp's conclusion is correct: empirical violation of Bell's inequalities demonstrates the non-locality of Nature, not 
merely of viable hidden-variable theories. Thus, another way to frame the goal of the present paper is this: we give an alternative to Stapp's (failed) attempt to prove this shared conclusion.

The argument to be presented (and it is really just a detailed fleshing-out of the argument hinted at by Bell) is different from Stapp's in several important ways. First, instead of trying to arrive at an empirically testable inequality (or a logical contradiction) without assuming the existence of hidden variables, we show that hidden variables (of a certain specific character to be identified) must exist under the assumption of locality. Then, it is non-controversial to point out that Bell's Theorem (in its original form) renders any hidden-variables theory of this type inconsistent with experiment. Our argument is thus not a different ("stronger") version of Bell's Theorem; it is not a "Bell's Theorem without hidden-variables." 20] Rather, it is a proof that the hidden-variable and locality assumptions needed to derive Bell's Inequality are not on an equal logical footing in the overall argument. The hidden variables posited by Bell are not an "ad hoc assumption" but, rather, a logical implication of locality.

Second, our locality criterion differs from the various senses of locality utilized by Stapp. As mentioned, we present and then use the straightforward mathematical local causality condition introduced and advocated by J. S. Bell: "Bell Locality."

Third, where Stapp (and his critics) focus on specific (possible) measurement outcomes, our emphasis is instead on theories. In particular, the question from which our derivation proceeds is: What properties must a theory possess if it is to satisfy Bell Locality and agree with certain empirical facts? This emphasis on the structure of theories will be seen to follow in a natural way from Bell's locality condition, elaborated in Section 1 .

The upshot of these differences is that our proof is immune to the kinds of objections given against Stapp by (for example) Mermin, Unruh, Shimony and Stein. The argument presented here thus supplements Bell's Theorem in just the way needed to conclusively establish the following (Stapp-like) conclusion: no Bell Local theory can be consistent with the predictions of quantum mechanics, i.e., with the empirical facts. Note in particular that the conclusion is not restricted to hidden-variable theories, deterministic theories, or any other class. It applies to all theories, orthodox quantum theory very much included. Finally, given that no Bell Local theory can be empirically viable, it follows that Nature does not respect Bell Locality. This is the conclusion that is meant to be conveyed by the paper's title.

\section{BELL LOCALITY}

Perhaps the single greatest source of confusion over what, exactly, Bell's Theorem proves is confusion and ambiguity over the meaning of "locality." Many different senses of locality have been defined in the literature.
[3, 21] Our purpose here is not to review this literature, nor to present a conclusive argument for the appropriateness of any specific definition. Rather, we will simply pick a particular definition of locality and show that an interesting conclusion follows from it.

The one in question is (what we shall call) Bell Locality. This is essentially the locality criterion that was used, ever since his initial 1964 paper, in Bell's derivations of the famous inequality. His most detailed discussion of this locality concept may be found in the article "La Nouvelle Cuisine." [1, pages 232-48] Here we shall simply state the condition and then make a few clarifying remarks.

The following is Bell's description of the locality condition, along with his accompanying figure:

"A theory will be said to be locally causal [i.e., what we are calling Bell Local] if the probabilities attached to values of local beables in a space-time region 1 are unaltered by specification of values of local beables in a space-like separated region 2 , when what happens in the backward light cone of 1 is already sufficiently specified, for example by a full specification of local beables in a spacetime region 3." [1, page 240]

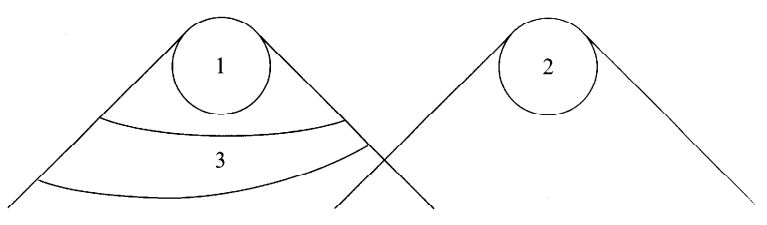

FIG. 1: "Full specification of what happens in 3 makes events in 2 irrelevant for predictions about 1 in a locally causal [i.e., Bell Local] theory." (Figure and caption are from [1, pg. 240].)

As Bell explains, "It is important that region 3 completely shields off from 1 the overlap of the backward light cones of 1 and 2 . Otherwise the traces in region 2 of causes of events in 1 could well supplement whatever else was being used for calculating probabilities about 1 . The hypothesis is that any such information about 2 becomes redundant when 3 is specified completely." 1, pg. $240]$

Let us now apply this to the standard EPR-Bell setup in which a particle source emits pairs of oppositelydirected neutral spin-1/2 particles in (what orthodox quantum theory would describe as) a spin singlet state. Two experimenters, Alice and Bob, are located at some distance from the source; each possesses a Stern-Gerlach type device which allows them to measure the component of the spin of their particles (along, respectively, the directions $\hat{a}$ and $\hat{b}$ ), yielding outcomes $A= \pm 1$ and $B= \pm 1$. Note that we consider $\hat{a}$ and $\hat{b}$ to be free variables - not in any way affecting, dependent on, or correlated with 


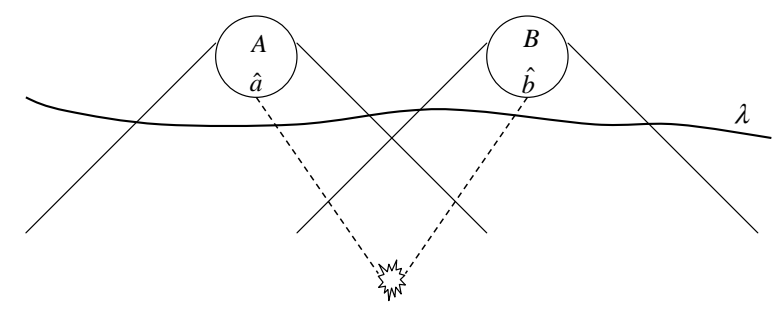

FIG. 2: A pair of neutral spin-1/2 particles is created, with the individual particles heading to the left and right, where Alice and Bob freely choose an axis (respectively $\hat{a}$ and $\hat{b}$ ) along which to measure their particle's spin component. The outcomes of those experiments are given by $A= \pm 1$ and $B= \pm 1$. The symbol $\lambda$ denotes a complete specification of the state of the particle pair (and, if necessary, the pre-measurement state of the detection apparatuses) across some space-like hypersurface satisfying (for both measurement events) the constraints Bell describes for his region 3 from Figure 1.

one another or with the prior state of the particle pair, whose complete description on the space-like hypersurface indicated in Figure 2 we denote $\lambda$. (In principle, a theory might assert that the outcome of the experiments depends not just on the settings $\hat{a}$ and $\hat{b}$ and the state of the particle pair, but also on some other facts about the measuring apparatus; we may always include such facts in our definition of $\lambda$ without affecting the argument. Thus, throughout the following, the phrase "state of the particle pair" should be read as "state of the particle pair, detection apparatuses, and anything else - excluding of course $\hat{a}$ and $\hat{b}$ - upon which the theory in question claims the outcomes depend.") We shall also assume that the space-time regions of Alice's and Bob's measurements (indicated by the circles in Figure 2) are space-like separated (as shown in the Figure).

Bell Locality then entails the following: once we specify a complete description of the pre-measurement state of the particle pair, the probability for Alice to obtain a certain outcome $A$ for a measurement along a certain direction $\hat{a}$ is independent of the setting $(\hat{b})$ and outcome $(B)$ of Bob's experiment. In particular, the probability in question does not change depending on whether we do or do not specify this information about Bob's experiment. In Bell's words, that information about the distant experiment "becomes redundant" when the state of the particle pair is specified completely. Formally:

$$
P(A \mid \hat{a}, \hat{b}, B, \lambda)=P(A \mid \hat{a}, \lambda)
$$

where $P(X \mid Y)$ means the conditional probability of $X$ given $Y$. Bell Locality of course also requires the same condition for Bob's outcomes:

$$
P(B \mid \hat{a}, \hat{b}, A, \lambda)=P(B \mid \hat{b}, \lambda) .
$$

There are several key points here:
- The symbol $\lambda$ appearing in the definition of Bell Locality refers to a complete description of the state of the particle pair (and, as needed, facts about the experimental apparatuses - other than the freelychoosable settings - on which the probabilities may depend) along the space-like hypersurface shown in Figure 2 .

- In principle, only some particular candidate theory can tell us what $\lambda$ consists of. The probabilities about which Bell Locality speaks thus refer not to empirical frequencies of given outcomes, but rather to probabilities as assigned by the fundamental dynamics of some particular theory - namely, whatever theory posits $\lambda$ as a complete state description. Thus, in the primary sense, what Bell Locality constrains is theories. Bell himself stressed this point in "Free variables and local causality" 1, pg 100-104]. Here is the relevant passage: "I would insist here on the distinction between analyzing various physical theories, on the one hand, and philosophising about the unique real world on the other hand. In this matter of causality it is a great inconvenience that the real world is given to us once only. We cannot know what would have happened if something had been different. We cannot repeat an experiment changing just one variable; the hands of the clock will have moved, and the moons of Jupiter. Physical theories are more amenable in this respect. We can calculate the consequences of changing free elements in a theory, be they only initial conditions, and so can explore the causal structure of the theory. I insist that [my local causality criterion] is primarily an analysis of certain kinds of physical theory." Note also that Bell's characterization of the locality condition (the first block-quote in this section) begins: "A theory will be said to be locally causal if..." [emphasis added]. Given some candidate theory, we may ask: Does this theory respect Eq. (10), or not? That is: Is this theory Bell Local, or not? This is the source of the theory-emphasis (as opposed to experimental-outcome-emphasis) mentioned in the introduction.

- Given this need to focus on theories, it is necessary to clarify what we mean by a theory. We will stipulate just what is required to permit an unambiguous determination of whether or not a theory satisfies Bell Locality. Thus: a theory must provide some particular account of $\lambda$. It must tell us what a complete description of the state of the particle pair consists of. And it must provide, on that basis, a definite formal structure by which the probabilities for the relevant possible experimental outcomes can be calculated.

One important logical implication of Bell Locality is that it requires joint probabilities for space-like separated 
events to factorize when $\lambda$ is specified:

$$
\begin{aligned}
P(A, B \mid \hat{a}, \hat{b}, \lambda) & =P(A \mid B, \hat{a}, \hat{b}, \lambda) \times P(B \mid \hat{a}, \hat{b}, \lambda) \\
& =P(A \mid \hat{a}, \lambda) \times P(B \mid \hat{b}, \lambda) .
\end{aligned}
$$

The first line merely expresses the joint probability in terms of a conditional probability, while the move to the second line involves a trivial application of Eqs. (11) and (2). As Bell remarks, "Very often such factorizability is taken as the starting point of the analysis. Here we have preferred to see it not as the formulation of "local causality', but as a consequence thereof." [1, pg 243]

In principle, nothing more about Bell Locality need be said. It is a straightforward mathematical condition that permits an unambiguous answer to the question: Is Theory X Bell Local? However, some additional remarks may help clarify the condition and motivate the subsequent derivation.

Bell intended this mathematical locality condition as a precise statement of relativity's prohibition on superluminal causation. The idea here is that the causes of a given event should be located exclusively in that event's past light cone - i.e., a complete specification of physical states in the past light cone should be sufficient to uniquely and finally define the probability of the event in question (such that specification of additional information from a space-like separated region is redundant) - i.e., the probability attributed by the theory in question to that event should depend only on facts in the event's past light cone (and not on facts outside the past light cone). As Bell discusses in "The theory of local beables" [1, pg 52-62] this is a natural extension of the obvious definition of local causality for deterministic theories (namely, the particular outcome assigned by the theory should depend only on facts in the past light cone) to theories which are not deterministic, which are irreducibly stochastic. Such theories replace definite predictions for which outcome will occur, with definite predictions for the probabilities of various possible outcomes. Bell Locality is then simply the same requirement for those probabilities (namely, that they not depend on facts outside the past light cone) that the obvious definition of locality for deterministic theories imposes on those theories' outcome predictions.

Since there has been such confusion about this in the literature, let us elaborate a bit more. Any causal dependence of a given event on other events outside the past light cone would involve superluminal (or reversetemporal) influence - something that is supposed to be ruled out by the space-time structure of relativity (and common-sense ideas about the meaning of cause and effect). Bell's idea was that the assertion of such stochastic dependence by a theory is tantamount to the assertion of a causal dependence (recall Bell's comments in the second bulleted point above). And so relativity's prohibition on superluminal causation requires that the probability assigned, by a theory, to a given event (Alice's outcome, say) shouldn't depend (according to the postulated dy- namics of that theory) on either the setting of Bob's apparatus $(\hat{b})$ or on the outcome of his experiment $(B)$ or anything else outside the past light cone of $A$. As Bell explains, such information should (for a relativistic theory) be redundant (or just irrelevant).

According to relativity, information pertaining to a space-like separated region ought to be causally irrelevant to the event in question. This does not necessarily mean, however, that such information must be statistically irrelevant at the level of relative frequencies of outcomes. Correlations between space-like separated events which are not directly causally related is certainly possible. 29] They could be correlated, for example, by virtue of their being both effects of some other, earlier shared cause. But such an earlier shared cause would evidently have to be included in our (by hypothesis) complete specification of the state of the particle pair, $\lambda$. For a given event (such as $A$ ), $\lambda$ already includes all the information (other than $\hat{a}$ ) that is (according to the theory in question) causally relevant to that event. And therefore, the probability assigned to that event under condition $\lambda$, will not (in a Bell Local theory) depend on additional information pertaining to a space-like separated region. The event in question may turn out to be correlated with some such additional information at the level of relative frequencies, but its probability should not depend on that information at the level of the fundamental theoretical dynamics.

To all appearances, Bell Locality seems to be exactly what is required by relativity's prohibition on superluminal causation. Any theory which violates Bell Locality necessarily posits a causal influence between space-like separated regions of space-time.

There is no real point in claiming that Bell Locality is the only possible reasonable mathematical specification of relativistic causality. We wish to stress, however, that Bell Locality is prima facie reasonable in this role. There is excellent reason to think that a genuinely relativistic theory must respect Bell Locality. From the standard point of view according to which relativity theory is accepted as an established fact, it would be a surprise to find that Bell Locality could not be maintained. Yet this is just what Bell's two-part argument establishes.

Before moving on to the proof of the first part of this argument, let us stress here several things which are not presupposed by Bell Locality. First, Bell Locality does not assume or require determinism; the condition is stated in terms of the probabilities assigned to various events by a given theory's dynamical laws. Determinism is of course included as a special case (in which all the probabilities assume exclusively the values one or zero), but we do not impose this as an assumption. Second, the formulation of Bell Locality in no way assumes or requires the existence of hidden-variables. The condition is stated in terms of a complete state description $(\lambda)$ for the particle pair, but there is nothing in the definition of Bell Locality which requires $\lambda$ to include anything more than the quantum mechanical wave function (which, ac- 
cording to orthodox quantum theory, provides already a complete description of the state of the particle pair). Indeed, it need not even include that. We make no assumptions at all about the content of $\lambda$, i.e., we in no way restrict the class of theories to which we are (initially) open. Bell Locality simply permits us to easily assess whether a given theory (of any type, so long as it satisfies the criteria stated above, i.e., so long as it proposes some definite candidate $\lambda$ and permits one to calculate probabilities on that basis) is or is not locally causal.

The twist, to be utilized in Section III is that we may infer something about the nature of $\lambda$ from the requirements that Bell Locality - and certain empiricallysupported facts - be respected.

\section{HIDDEN VARIABLES}

Having clarified the definition and meaning of Bell Locality, let us now turn to the main topic: What features must a Bell Local theory possess in order to account for certain empirically-observed facts?

The empirical fact that will concern us is the following: if the particle pair is prepared in (what orthodox quantum theory describes as) the spin singlet state

$$
|\psi\rangle=\frac{1}{\sqrt{2}}(|+1\rangle|-1\rangle-|-1\rangle|+1\rangle)
$$

and if Alice and Bob happen to choose to measure along the same axis $(\hat{a}=\hat{b})$, the outcomes will be perfectly anti-correlated: either $A=+1$ and $B=-1$, or $A=-1$ and $B=+1$. Can a Bell Local theory account for this empirical fact, and if so, how?

To begin, we must explain what is meant by "preparing the particle pair in the spin singlet state." What this means experimentally and operationally is clear, but what does it mean in terms of the (as yet completely unspecified) theory which will (we hope) be able to account for the results? Evidently the preparation procedure creates or selects one specific state out of a class of possible states consistent with the preparation procedure. Let us denote this class $\Lambda_{\psi}$. Thus, a particular instance of the preparation will result in some particular $\lambda \in \Lambda_{\psi}$. (We consider the set $\Lambda_{\psi}$ to consist of those states which can be produced with nonzero probability. Also note that we of course leave open the possibility that $\Lambda_{\psi}$ is a set with only one element, e.g., the wave function itself. That is, we leave open, for now, the possibility that the orthodox quantum state description is complete. Finally, note that we assume $\Lambda_{\psi}$ contains a finite number of elements. Everything that follows can be applied to the case of an infinite $\Lambda_{\psi}$, mutatis mutandis.)

Consider now the joint probability for outcomes $A=$ $+1, B=+1$ when $\hat{a}=\hat{b}=\hat{n}_{1}$. In a Bell Local theory, this joint probability must factor into a product as follows:

$$
P\left(A=+1, B=+1 \mid \hat{n}_{1}, \hat{n}_{1}, \lambda\right)
$$

$$
=P\left(A=+1 \mid \hat{n}_{1}, \lambda\right) \times P\left(B=+1 \mid \hat{n}_{1}, \lambda\right) .
$$

We are taking it as given (because it is known from experiment) that this joint probability vanishes for all $\lambda \in \Lambda_{\psi}$. If there were some $\lambda$ that could, with nonzero probability, be produced by the singlet preparation procedure, and for which the joint probability above were other than zero, Alice and Bob would sometimes get identical outcomes even though they measured along the same axis. Since in fact this never happens, we constrain the theories accordingly.

Thus $\forall \lambda \in \Lambda_{\psi}$, either

$$
P\left(A=+1 \mid \hat{n}_{1}, \lambda\right)=0
$$

or

$$
P\left(B=+1 \mid \hat{n}_{1}, \lambda\right)=0 .
$$

Now consider the joint probability for outcomes $A=$ $-1, B=-1$ when (again) Alice and Bob both measure along the direction $\hat{n}_{1}$. As above, we must have that $\forall \lambda \in \Lambda_{\psi}$ either

$$
P\left(A=-1 \mid \hat{n}_{1}, \lambda\right)=0
$$

or

$$
P\left(B=-1 \mid \hat{n}_{1}, \lambda\right)=0 .
$$

Note that the two conditions - (6) or (7), on the one hand, and, on the other, (8) or (9) - must be satisfied simultaneously for all $\lambda \in \Lambda_{\psi}$.

Further, for any particular $\lambda$, a statement of the form

$$
P\left(A=+1 \mid \hat{n}_{1}, \lambda\right)=0
$$

implies one of the form

$$
P\left(A=-1 \mid \hat{n}_{1}, \lambda\right)=1
$$

since the outcomes are bivalent: if, for a given $\lambda$ and a given measurement direction, a certain outcome is (according to some theory) impossible, then, since there are only two possible outcomes, the opposite outcome is required. Recall too that the probabilities here are not empirical relative frequencies, but probabilities as assigned by the fundamental dynamics of a theory. The assertion here is that if a given theory assigns zero probability to a certain outcome (under specified conditions), then it must assign unit probability to the (here only) alternative outcome. This suggests a shorthand notation in which we substitute, e.g., for Eq. (11) the simpler statement

$$
A\left(\hat{n}_{1}, \lambda\right)=-1
$$

and similarly for the other possibilities.

The reader may worry that we are here violating Wheeler's famous statement of the orthodox quantum philosophy: "No elementary phenomenon is a phenomenon until it is a registered (observed) phenomenon" 
22 - i.e., it is invalid to attribute particular outcomes to experiments which haven't, in fact, been performed. This worry is partly justified. We are not, however, asserting that an un-performed measurement has an actual, particular outcome; this would be literal nonsense, and is the grain of truth in Wheeler's dictum. Strictly speaking, our statement isn't even about Alice's measurement - it is about the state $\lambda$ and the theory in which that state assignment is embedded. The real meaning of Eq. (12) is simply this: for the state $\lambda$, the theory in question assigns unit probability to the outcome $A=-1$ under the condition that Alice measures along direction $\hat{n}_{1}$. The theory must attribute sufficient structure to $\lambda$ (and possess the necessary dynamical laws) such that, should Alice choose to measure along $\hat{n}_{1}$, the outcome $A=-1$ is guaranteed. In this sense, we may say that the theory in question encodes the outcome $A=-1$ (for measurement along $\hat{n}_{1}$ ) in the state $\lambda$.

Crucially, we must not forget that this kind of statement is simply forced on us by the logical development to this point: given that we only consider Bell Local theories, and given that the theories must be capable of making the correct predictions for the (possible) case $\hat{a}=\hat{b}=\hat{n}_{1}$, the theory must posit this sort of structure. Thus, to whatever extent the required theoretical structure conflicts with Wheeler's dictum, we must evidently conclude that this orthodox philosophy is excluded by our premises (Bell Locality and perfect anti-correlation).

Summarizing the results so far, we must have that, $\forall \lambda \in \Lambda_{\psi}$

$$
A\left(\hat{n}_{1}, \lambda\right)=-1 \quad \text { or } \quad B\left(\hat{n}_{1}, \lambda\right)=-1
$$

and

$$
A\left(\hat{n}_{1}, \lambda\right)=+1 \quad \text { or } \quad B\left(\hat{n}_{1}, \lambda\right)=+1 .
$$

Since we cannot have both $A\left(\hat{n}_{1}, \lambda\right)=-1$ and $A\left(\hat{n}_{1}, \lambda\right)=$ +1 for the same $\lambda$ (and likewise for $B$ ), Eqs. (13) and (14) are logically equivalent to the following: either

$$
A\left(\hat{n}_{1}, \lambda\right)=-1 \quad \text { and } \quad B\left(\hat{n}_{1}, \lambda\right)=+1
$$

or

$$
A\left(\hat{n}_{1}, \lambda\right)=+1 \quad \text { and } \quad B\left(\hat{n}_{1}, \lambda\right)=-1 .
$$

In other words, the requirements mentioned in Eqs. (6) - (9) will be satisfied if and only if the states $\lambda \in \Lambda_{\psi}$ divide into two mutually exclusive and jointly exhaustive classes: those (call them $\Lambda_{\psi}^{\hat{n}_{1}+-}$ ) for which $A\left(\hat{n}_{1}, \lambda\right)=+1$ and $B\left(\hat{n}_{1}, \lambda\right)=-1$; and those (call them $\Lambda_{\psi}^{\hat{n}_{1}-+}$ ) for which $A\left(\hat{n}_{1}, \lambda\right)=-1$ and $B\left(\hat{n}_{1}, \lambda\right)=+1$. The two classes $\left(\Lambda_{\psi}^{\hat{n}_{1}+-}\right.$ and $\left.\Lambda_{\psi}^{\hat{n}_{1}-+}\right)$ are jointly exhaustive because any $\lambda$ which, with nonzero probability, allows for identical outcomes on the two sides, will necessarily spoil the theory's ability to predict perfect anti-correlation. Any such $\lambda$ is by definition excluded from $\Lambda_{\psi}$ to begin with.

In a Bell Local theory, the probability for a given outcome on one side (conditionalized on a complete specification of the state of the particles) may not depend on goings-on at the distant location. Given the empirically observed fact of perfect anti-correlation, we have shown that a Bell Local theory must attribute outcomedetermining properties to the particle pair. Putting the same point another way, we have proved that there is no such thing as a Bell Local theory which accounts for the perfect anti-correlation without positing deterministic hidden variables ("hidden" because these variables evidently go beyond the quantum state description). All Bell Local theories which successfully predict perfect anti-correlation (under the appropriate circumstances) must posit that the individual outcomes are determined, in advance of the actual measurements, by structure encoded in $\lambda$. Notice too that the proof that this kind of theoretical structure is required (for a Bell Local theory) in no way assumes the actual performance of measurements along $\hat{a}=\hat{b}=\hat{n}_{1}$. The mere fact that such measurements may be later chosen - combined with the requirement that the theory must be capable of generating the appropriate sorts of outcomes should this eventuality arise - leads to the conditions described above.

A key point is now the generalizability of this result to measurements along different axes, say $\hat{n}_{2}$ and $\hat{n}_{3}$. Following exactly the logic of the above paragraphs, we may argue that the states $\lambda \in \Lambda_{\psi}$ must divide into classes $\Lambda_{\psi}^{\hat{n}_{2}+-}$ and $\Lambda_{\psi}^{\hat{n}_{2}-+}$, such that, respectively,

$$
A\left(\hat{n}_{2}, \lambda\right)=+1 \quad \text { and } \quad B\left(\hat{n}_{2}, \lambda\right)=-1
$$

and

$$
A\left(\hat{n}_{2}, \lambda\right)=-1 \quad \text { and } \quad B\left(\hat{n}_{2}, \lambda\right)=+1
$$

And similarly for $\hat{n}_{3}$.

Are we, however, going beyond what is required if we insist on dividing the class $\Lambda_{\psi}$ using the three measurement directions simultaneously? That is, are we permitted to deduce (from the fact of perfect anti-correlation along each of the three directions $\left\{\hat{n}_{1}, \hat{n}_{2}, \hat{n}_{3}\right\}$ and the requirement of Bell Locality) that a theory must posit eight mutually exclusive and jointly exhaustive sub-classes of $\Lambda_{\psi}$ - for example, the class $\Lambda_{\psi}^{\hat{n}_{1}+-, \hat{n}_{2}+-, \hat{n}_{3}+-}$ such that $\forall \lambda \in \Lambda_{\psi}^{\hat{n}_{1}+-, \hat{n}_{2}+-, \hat{n}_{3}+-}$

$$
\left\{\begin{array}{ll}
A\left(\hat{n}_{1}, \lambda\right)=+1, & B\left(\hat{n}_{1}, \lambda\right)=-1 \\
A\left(\hat{n}_{2}, \lambda\right)=+1, & B\left(\hat{n}_{2}, \lambda\right)=-1 \\
A\left(\hat{n}_{3}, \lambda\right)=+1, & B\left(\hat{n}_{3}, \lambda\right)=-1
\end{array}\right\}
$$

and similarly for the other seven possible combinations? This is of course tantamount to assigning premeasurement values to the spin components of each particle along all three considered measurement directions (and, in principle, along the continuous infinity of possible measurement directions).

Such an assignment is obviously in conflict with the orthodox quantum philosophy, which expressly forbids assigning values to unmeasured observables (not to mention assigning values simultaneously to non-commuting 
observables!). But that is not the relevant issue. What concerns us here is simply this: is such an assignment required by Bell Locality? That is, must a Bell Local theory predicting perfect anti-correlation (along all of the three considered measurement axes) posit this detailed structure in the state descriptions?

The answer is unambiguously yes. A Bell Local theory (which predicts successfully the perfect anti-correlation) must posit this structure, must posit enough detailed content to $\lambda$ that the outcome of the measurement of any spin component on either particle is determined, as, for example, in Eq. (19). The reason is simple: we are treating the measurement axes $\hat{a}$ and $\hat{b}$ as free variables which can, in principle, be set randomly (or by a free-will choice) after the pair's state $\lambda$ is fixed. Thus, by precisely the reasoning detailed above, the states $\lambda \in \Lambda_{\psi}$ must be so as to produce perfect anti-correlation should Alice and Bob happen to both measure along any of the three considered directions. This requirement alone does not necessitate the kind of detailed structure contained in Eq. (19). When combined with the requirement of Bell Locality, however, this detailed structure - these deterministic hidden variables - are required. A theory (like orthodox quantum mechanics) which posits less structure for $\lambda$ can successfully predict perfect anti-correlation along any of the three considered directions - but only at the price of violating Bell Locality.

\section{DISCUSSION}

The main result proved in Section [III is the following: All theories respecting a certain locality condition (Bell Locality) must, in order to successfully reproduce a certain class of empirically well-confirmed correlations, posit that the outcomes of all possible spin-component measurements to be made on the particles are encoded in the pre-measurement state of the particles, such that the outcome in one wing of the experiment is determined, once the state of the particle pair and the orientation of the nearby apparatus are specified. 23] In rough terms, the particles must carry "instruction sets" 24] which predetermine the outcomes of spin measurements. Since including such "instruction sets" in the state specification goes beyond what is attributed to the states by orthodox quantum theory, the kind of theory we have argued for may be termed a hidden-variable theory. What is crucial here is that the relevant hidden variables have not been assumed, but rather derived - in particular, derived from the logical conjunction of (1) a certain class of empirically supported correlations and (2) Bell Locality.

It is of course well known that a hidden-variable theory of this type cannot account for another class of empirically tested correlations, and is therefore not empirically viable. 25] This is Bell's Theorem. It would be a logical fallacy, however, to conclude from this that the arguments presented in Section IIII are flawed. This would involve an equivocation between the truth of the con- clusion of an argument, and the validity of the argument itself. What we have established here is that hidden variables of a particular variety are required if one insists on respecting Bell Locality. Since such hidden variable theories are evidently not viable, the proper conclusion to draw is that Bell Locality cannot be maintained - i.e., no theory respecting Bell Locality can account for the entire class of empirically observed correlations between distant particles.

The logic here was expressed clearly by Bell:
"The EPRB correlations are such that the result of the experiment on one side imme- diately foretells that on the other, whenever the analyzers happen to be parallel. If we do not accept the intervention on one side as a causal influence on the other, we seem obliged to admit that the results on both sides are de- termined in advance anyway, independently of the intervention on the other side, by sig- nals from the source and by the local magnet setting. But this has implications for the non- parallel settings which conflict with those of quantum mechanics. So we cannot dismiss intervention on one side as a causal influence on the other." [1, pg 149-50]

The same basic argument has also been advocated by a number of other authors, for example those in the first of the "camps" described in Section \ The present paper adds two notable features: first, a precise mathematical clarification of Bell's phrase "if we do not accept the intervention on one side as a causal influence on the other" and, second, a rigorous mathematical derivation of what Bell merely says "we seem obliged to admit."

H. P. Stapp apparently agrees with Bell that one "cannot dismiss intervention on one side as a causal influence on the other." But, as discussed in the introduction, Stapp's argument for this conclusion is different from that advocated by Bell. And since Stapp's arguments have been shown to suffer from several apparently fatal flaws, it is worth clarifying in some detail how Bell's argument (elaborated and sharpened in the present paper) differs from Stapp's.

First, let us emphasize the point about overall logical structure. Stapp has attempted to prove a "Belltype theorem without hidden variables" - i.e., to prove a stronger version of Bell's Theorem which dispenses with one of the premises on which Bell's own derivation rests. By contrast, we retain the original ("weak") version of Bell's Theorem, and supplement it by showing that the two premises used in deriving the inequality - (i) locality and (ii) hidden variables - are not logically unrelated axioms. Rather, premise (ii) follows from premise (i) and the empirical fact of perfect anti-correlation.

The criticisms made against Stapp by Mermin, Unruh, Shimony, Stein, and others suggest that Bell's Theorem cannot be strengthened in the way that would be required to validate Stapp's route to our shared conclusion. One 
simply cannot arrive at a Bell Inequality (or the corresponding logical contradiction arrived at in the context of Hardy's Theorem) if one rules out any assumption of hidden-variables or counterfactual-definiteness and thus restricts the discussion to actual measurement results.

The reason such a restriction precludes a valid nonlocality proof is straightforward. In any particular run of the experiment, only one measurement can actually be performed in each wing. There are, thus, two and only two actual measurement outcomes to work with. But any such pair of outcomes can always and trivially be accounted for locally - namely, by simply asserting that the measurements revealed locally pre-existing values for the observables in question. Of course, one is then no longer talking about orthodox quantum theory but, rather, about some kind of local hidden-variable theory. But if one has decided to focus exclusively on measurement outcomes (and not on the specific theories which predict those outcomes), this is a distinction without a difference and no nonlocality can be established.

One might also allow talk of the relative frequencies with which various outcomes appear. It would then be possible to define locality by the insistence that the relative frequency of a given outcome on one side (say, $B$ ) not correlate with the freely chosen setting $(\hat{a})$ on the other side. [30] In our terminology, this condition would read

$$
P(B \mid \hat{a}, \hat{b})=P\left(B \mid \hat{a}^{\prime}, \hat{b}\right)
$$

where

$$
P(B \mid \hat{a}, \hat{b})=\sum_{\lambda \in \Lambda_{\psi}} \sum_{A= \pm 1} P(A, B \mid \hat{a}, \hat{b}, \lambda) P_{\psi}(\lambda),
$$

$\hat{a}$ and $\hat{a}^{\prime}$ are two different possible (freely-choosable) settings of the apparatus on the left, and $P_{\psi}(\lambda)$ is the probability that the preparation procedure produces the particular state $\lambda$. This condition is called Signal Locality because its violation would permit Alice to send superluminal signals to Bob. It is well known that orthodox quantum theory does not violate Signal Locality. 26 Moreover, since the condition refers only to relative frequencies of outcomes, all theories which share the same empirical predictions as orthodox quantum theory (for example, Bohmian Mechanics) also respect Signal Locality.

Either way, so long as one restricts one's analysis to exclusively observable phenomena, there is no hope of establishing nonlocality. And this is the deepest reason that Stapp's project fails. In his original paper, Stapp defines locality as follows: "no free choice can influence observable phenomena lying outside its forward light cone". 12. But for the reasons just indicated, this definition (which is apparently equivalent to Signal Locality) is too restrictive. It is impossible to establish the reality of a violation of this sort of locality.

Stapp attempts to get around this difficulty by using, in his actual proof, a less restrictive definition of locality.
(Thus, he regards the fact that the truth of his statement $S$ depends on a distant free choice, as establishing a violation of locality even though $S$ - a counterfactual conditional - is not an "observable phenomenon".) And this stronger definition of locality is precisely what his critics have criticized: the stronger definition "can be interpreted as assigning an unwarranted level of reality to the value of certain quantum attributes." [15] That is, it doesn't comport with the positivist or phenomenalist approach of the orthodox quantum philosophy.

Stapp repeatedly stresses that "all the assumptions used in [his] proof are elements of orthodox quantum philosophy". 27] At root, it is precisely Stapp's allegiance to this philosophy which prevents him from constructing a valid proof. The conclusion he wants to establish (which is about causality, not merely the ability to send signals) is, according to that positivist philosophy, off the table from the very beginning.

The phenomenalist attitude of the orthodox quantum philosophy also invites the identification of quantum theory with any theory sharing its empirical predictions. 31] Stapp's acceptance of this philosophy then leads to a recurring confusion between two different goals: showing that quantum theory itself is nonlocal, and showing that any theory sharing quantum theory's empirical predictions must be nonlocal. For example, despite apparently attempting to show that some sort of nonlocality is required by the quantum mechanical predictions (i.e., required of any theory which shares those predictions), Stapp titles his paper "Nonlocal character of quantum theory."

In fact, though, establishing the nonlocal character of orthodox quantum theory is easy: once (an appropriate sense of) locality is defined precisely (as in Section [II), it is a trivial observation that the orthodox theory (where $\lambda$ is simply $\psi$ ) fails to respect Eq. (3) and hence is not local. The difficult assignment is to show not just that orthodox quantum theory is nonlocal, but that this nonlocality is ineliminable - that no Bell Local theory can share the quantum mechanical predictions (or, equivalently, can match experiment). Stapp's allegiance to the orthodox quantum philosophy thus ensures in advance that his project cannot succeed: when he inevitably slides over from the easy assignment to the hard assignment, he necessarily goes beyond standard quantum theory and thus leaves himself open to the objection that he is "assigning an unwarranted level of reality to" certain things. Given his expressed premises, this objection is unavoidable and completely fatal.

Stapp once described his project as follows: "The nonlocality that I claim to exhibit is completely compatible with the locality properties of relativity theory, which, in a quantum context, pertain only to features of our observations, not to features of a putative underlying reality." 27. Since the empirical predictions of quantum theory respect Signal Locality, there is no way to "exhibit" any nonlocality at the level of "our observations." It simply cannot be done. But if, motivated by the orthodox quan- 
tum philosophy, one excludes from the beginning any talk about the "features of a putative underlying reality", then there is literally nothing else - that is, no other sense of locality - to discuss. The vague anti-realism of the orthodox quantum philosophy thus seems to rule out the very kind of talk that is absolutely required to show that nature violates some locality condition - namely, talk of nature! But orthodox quantum theory better commit to a realistic description of something. Otherwise that is, if one retreats to an exclusively epistemological or "algorithmic" interpretation of quantum theory in general and the wave function in particular - one simply no longer has a theory in the sense defined in Section III It is then meaningless to discuss whether the causal processes posited by the "theory" respect relativity's prohibitions on superluminal causation. A formalism which is not about any such processes is neither local nor nonlocal. Both terms are simply inapplicable. 28]

One can start to see the shiftiness of the orthodox quantum philosophy which led Bell to describe it as "unprofessionally vague and ambiguous." 1, pg 173] Ultimately, it is Stapp's acceptance of this philosophy which undercuts his attempt to exhibit nonlocality. Specifically, it prevents him (a) from articulating a clear definition of local causality, (b) from clearly noting the nonlocality that is already present in the orthodox theory, and (c) from even considering the types of theories that might have been thought to have provided a local explanation of the correlations in question.

In the alternative to Stapp's approach advocated by Bell and elaborated here, we begin not with an arbitrary allegiance to the orthodox quantum philosophy, but with a straightforward, mathematically precise definition of local causality, motivated exclusively by relativity theory. We then ask: is there a theory which respects this locality condition and which is consistent with what we know from experiment about the correlations between distant particles (prepared in a certain way)? The answer turns out to be no. To see this, it is helpful to break the question into two stages:

1. What structure must a theory have if it is to respect the locality condition and successfully match a certain set of empirical facts? The precise answer to this question was provided in Section [II] above.

2. Is a theory with this structure consistent with all of the other empirical facts? The answer to this question is given by Bell's Theorem in its original version: no.
The main strength of this two-step approach is simply that it permits an interesting, yet unambiguous, conclusion: No Bell Local theory can be empirically viable.

Of course, one could always assert that the conclusion isn't, appearances to the contrary notwithstanding, all that interesting, since Bell Locality isn't a correct formalization of relativity's prohibition on superluminal causation. One possible argument for this view would be that orthodox quantum theory violates Bell Locality: if one insists on remaining faithful to the orthodox philosophy (and ensuring that quantum theory doesn't turn out to be nonlocal) then Bell Locality can't be the "right" definition of locality.

That argument is too obviously question-begging to deserve attention. Is there some more reasonable objection that could be given against the apparent appropriateness of Bell Locality? Perhaps. But Bell's case elaborated in Section Iabove - is, on its face, sufficiently plausible that the burden of proof should lie with those who reject this locality condition. If Bell Locality isn't a good formalization of "relativistic causality", why not? And what is?

Until and unless such questions can be answered, we must evidently agree with Bell that there exists an "incompatibility, at the deepest level, between the two fundamental pillars of contemporary theory..." [1, pg 172] No Bell Local theory can account for the empirically verified predictions of quantum theory. Nature is not Bell Local.

And, to return to the question of the hidden variables program with which we began, this surely means that the use of Bell's Theorem against Bohmian Mechanics (by the "second camp") is fundamentally misguided. Orthodox quantum theory itself violates Bell Locality, so the fact that hidden variable theories like Bohm's violate it as well is no argument in support of the standard theory. There simply is no Bell Local theory that is in agreement with experiment, so it is ridiculous to reject any one particular theory (which does agree with experiment!) merely on the grounds that it violates Bell Locality. Even leaving aside the question of the appropriateness of Bell Locality (as a formalization of relativistic causality), it is an inescapable (just, someone might conceivably argue, uninteresting) fact that the failure of Bell Locality is a real feature of the world. So it can hardly be a valid objection against Bohmian Mechanics (or any other theory) that it displays this feature.
[1] J. S. Bell, Speakable and Unspeakable in Quantum Mechanics (Second Edition), (Cambridge University Press, Cambridge (UK), 2004)

[2] H. P. Stapp, "Bell's Theorem and World Process," Nuovo Cimento 29B, 270-6, (1975)
[3] T. Maudlin, Quantum Non-Locality and Relativity (Second Edition), (Blackwell, Cambridge (MA), 2002)

[4] D. Dürr, N. Zanghi, and S. Goldstein, "Quantum Equilibrium and the Role of Operators as Observables in Quantum Theory," Journal of Statistical Physics 116, 959- 
1055, (2004). (See in particular Section 8: Hidden Variables.)

[5] T. Norsen, "EPR and Bell Locality," quant-ph/0408105 to appear in Are there Quantum Jumps? and On the Present Status of Quantum Mechanics, A. Bassi, D. Dür, T. Weber, and N. Zanghi, eds., AIP Conference Proceedings, 2006

[6] H. M. Wiseman, "From Einstein's Theorem to Bell's Theorem: A History of Quantum Nonlocality," Contemporary Physics 47, 79-88 (2006)

[7] E. Wigner, "Interpretation of Quantum Mechanics," (1976), reprinted in Quantum Theory and Measurement, J. A. Wheeler and W. H. Zurek, eds. (Princeton University Press, Princeton, 1983)

[8] N. David Mermin, "Hidden Variables and the Two Theorems of John Bell," Reviews of Modern Physics 65, 803815, (July 1993)

[9] Einstein, Podolsky, and Rosen, "Can quantum- mechanical description of physical reality be considered complete?" Physical Review 47, 777-780, (1935)

[10] S. Goldstein, "Bohmian Mechanics," The Stanford Encyclopedia of Philosophy, Edward N. Zalta (ed.), http://plato.stanford.edu/entries/qm-bohm see also R. Tumulka, "Understanding Bohmian mechanics: a dialogue," American Journal of Physics 72, 1220-6, (2004)

[11] H. P. Stapp, "Bell's Theorem Without Hidden Variables," quant-ph/0010047

[12] H. P. Stapp, "Nonlocal character of quantum theory," American Journal of Physics 65, 300-304, (1997)

[13] H. P. Stapp, "A Bell-type theorem without hidden variables," American Journal of Physics 72, 30-33, (2004)

[14] Lucien Hardy, "Quantum mechanics, local realistic theories, and Lorentz invariant realistic theories," Physical Review Letters 68, 2981-2984, (1992)

[15] W. Unruh, "Is Quantum Mechanics Non-Local?" Physical Review A 59, 126-130, (1999)

[16] A. Shimony, "An Analysis of Stapp's 'A Bell-type theorem without hidden variables'," quant-ph/0404121

[17] A. Shimony and H. Stein, "Comment on "Nonlocal character of quantum theory,'...," American Journal of Physics 69, 848-853, (2001)

[18] N. David Mermin, "Nonlocal character of quantum theory?" American Journal of Physics 66, 920-4, (1998)

[19] N. David Mermin, "Nonlocality and Bohr's reply to EPR," quant-ph/9712003

[20] H. P. Stapp, "Bell's Theorem Without Hidden Variables," op cit.; See also P. Eberhard, "Bell's Theorem Without Hidden Variables," Nuovo Cimento 38B, 75-80, (1977)

[21] P. Eberhard, "Bell's Theorem and the different concepts of locality," Nuovo Cimento 46B, 392-419, (1978)

[22] J. A. Wheeler, "Law Without Law" in J. A. Wheeler and W. H. Zurek, eds., Quantum Theory and Measurement (Princeton University Press, Princeton, 1983)

[23] Thanks to Arthur Fine for, after reading an initial draft of the current paper, pointing out to me that similar arguments have appeared previously in the literature: e.g., Brian Skyrms, "Counterfactual Definiteness and Local Causation," Philosophy of Science 49, 43-50, (1982); Patrick Suppes, "Some remarks on hidden variables and the EPR paradox," Erkenntnis 16, 311-314, (1981) (and references therein).

[24] N. D. Mermin, "Bringing home the atomic world: Quantum mysteries for anybody," American Journal of Physics 49, 940-943, (1981)

[25] G. Weihs, T. Jennewein, C. Simon, H. Weinfurter, and A. Zeilinger, "Violation of Bell's inequality under strict Einstein locality conditions," Physical Review Letters 81, 5039-5043, (1998)

[26] L. Ballentine and J. Jarrett, "Bell's theorem: does quantum mechanics contradict relativity?" American Journal of Physics 55, 696-701, (1987)

[27] H. P. Stapp, "Response to 'Comment on "Nonlocal character of quantum theory" ' by Abner Shimony and Howard Stein ...," American Journal of Physics 69, 8549, (2001)

[28] For some additional discussion of this point, see: T. Norsen, "Einstein's Boxes," American Journal of Physics 73, 164-176, (2005)

[29] Note that this is simply the denial of the contrapositive of the well-known fallacy: "correlation implies causality." If correlation does not imply a direct causal relation between two variables, then neither does the absence of a direct causal relation imply that the variables must be uncorrelated.

[30] Of course, one could also ask whether the relative frequency of a given outcome on one side $(B)$ correlates with the outcome $(A)$ on the far side. But, as explained in Section II such a correlation does not necessarily entail any nonlocal causation. Thus again, as long as one arbitrarily restricts one's attention to measurement results rather than the structure of the theories which predict those results, nonlocal causation cannot be proved (or even, really, clearly defined). On a related point, this seems to be the reason that otherwise sensible people came to accept that "parameter dependence" was relativistically excluded, while "outcome dependence" was consistent with relativity. If one identifies probabilities with relative frequencies, a violation of "parameter independence" (plus some other conditions) permits superluminal signaling (which is clearly outlawed by relativity) while a violation of "outcome independence" does not. But if one is speaking not of relative frequencies but of the fundamental dynamics of a theory, then any dependence of probabilities on space-like separated events should obviously be excluded on relativistic grounds. This is why Bell Locality (as opposed to any of the various weaker conditions into which it can in principle be analyzed) is such a reasonable formalization of relativity's prohibition on superluminal causation.

[31] This conflation already arose above, when it was pointed out that the "obvious and trivial" local explanation for a given pair of outcomes involves a rejection of the orthodox completeness doctrine. On the phenomenalist premise, however, the orthodox theory and the local hidden-variable theory are literally the same theory, and it becomes impossible to sort out which version is and which version isn't local. Note also that this phenomenalist identification of a theory with its empirical predictions renders the orthodox completeness claim literally meaningless. 